
\title{
25 Research Suare \\ The Utility of Telemedicine In Managing Patients After COVID 19
}

Krystian Bartczak ( $\square$ krystian.bartczak@umed.lodz.pl )

Medical University of Lodz

Joanna Milkowska-Dymanowska

Medical University of Lodz

Wojciech J. Piotrowski

Medical University of Lodz

Adam J. Bialas

Medical University of Lodz

\section{Research Article}

Keywords: COVID 19, long COVID, post-COVID recovery, telemedicine, telemonitoring, pandemic

Posted Date: February 9th, 2022

DOI: https://doi.org/10.21203/rs.3.rs-1293204/v1

License: (c) (1) This work is licensed under a Creative Commons Attribution 4.0 International License. Read Full License 


\section{Abstract}

1. Introduction: Despite growing knowledge about transmission and relatively wide access to prophylaxis, the world is still facing a severe acute respiratory syndrome coronavirus 2 (SARS CoV 2) global pandemic. Under these circumstances telemedicine emerges as a powerful tool for safe at-home surveillance after a hospital discharge; the data on when to safely release a patient after acute COVID 19 is scarce. Reckoning an urgent need for improving outpatient management and possibly fatal complications of the post-COVID period, we performed the pilot telemonitoring program described below.

2. Materials and methods: Patients were involved in the study between December 2020 and May 2021. Recruitment was performed either during the hospital discharge (those hospitalized in a Barlicki Memorial Hospital in Lodz) or first outpatient visit up to 6 weeks after discharge from another center. Every participant received equipment for daily saturation and heart rate measurement coupled with a tablet for remote data transmission. The measurements were made after at least fifteen minutes of rest in a sitting position without oxygen supplementation. Along with the measurements, the cough and dyspnea daily surveys ( 1 to 5 points) and Fatigue Assessment Scale weekly surveys were filled. We expected a drop of saturation during thromboembolic events, infectious complications, etc.

3. Results: A total of 30 patients were monitored for a minimum period of 45 days, at least 2 weeks after spontaneous saturation normalization. Mean age was 55 (mean 55.23; SD +/- 10.64 years). The group was divided according to clinical improvement defined as the $\geq 10 \%$ functional vital capacity (FVC) raise or $\geq 15 \%$ lung transfer for carbon monoxide $\left(T_{L, C O}\right)$ rise. Our findings suggest that at-rest home saturation measurements below $94 \%(p=0.03)$ correspond with the lack of clinical improvement in post-COVID observation $(\mathrm{p}=0.03)$. The non-improvement group presented with lower mean - 94 (93-96) \% vs 96 (95$97) \%, p=0.01$ and minimum saturation $-89(86-92) \%$ vs. $92(90-94) \%, p=0.04$. They also presented higher variations in saturation measurements; saturation amplitude was $9(7-11) \%$ vs $7(4-8) \%, p=0.03$; up to day 22 most of the saturation differences reached statistical significance. Last but not least, we discovered that participants missing 2 or more measurements during the observation were more often ranked into the clinical improvement group $(p=0.01)$. Heart rate day-to-day measurements did not differ between both groups; gathered data about dyspnea and cough intensity did not reach statistical significance either.

4. Conclusions: A better understanding of the disease's natural history will ultimately lead us to a better understanding of long COVID symptoms and corresponding threats. In this paper, we have found home oxygen saturation telemonitoring to be useful in the prediction of the trajectory of the disease course. Our findings suggest that detection of at-rest home saturation measurement equal to or below $94 \%$ corresponds with the lack of clinical improvement in the time of observation and this group of patients presented higher variability of day-to-day oxygen saturation measurements. The determination of which patient should be involved in telemedicine programs after discharge requests further research.

\section{Introduction}


Despite growing knowledge about transmission and relatively wide access to prophylaxis, the world is still facing a severe acute respiratory syndrome coronavirus 2 (SARS CoV 2) global pandemic. With every coronavirus disease 2019 (COVID 19) outbreak the hospitals are filling with worsening patients and it forces the already stable and treated individuals out of the hospital; some of them still suffering from hypoxemia and requiring further oxygen treatment. The need for optimization of resources has been noticed both by medics and politicians, while telemedicine can be a powerful tool for safe at-home treatment [1].

The knowledge about symptoms and natural history after discharge from COVID-19 is continuously evolving [2]. Currently, available research usually assessed patients in a defined schedule after hospital discharge though there is little information about the patients' condition between discharge and further outpatient assessment [3]. As far as we are concerned, there is no evidence-based data on when to safely release a patient from the hospital[4].

Telemedicine is defined as "the use of electronic information and communications technologies to provide and support health care when distance separates the participants" [5]. Telehealth measures are proven to be effective in controlling the epidemic, as well as improving patients' safety and treatment, especially those living in remote regions [6]. Reckoning an urgent need for improving patients' outpatient management and possibly fatal complications after hospital discharge, we started the pilot telemonitoring program described below.

\section{Methods}

This prospective study was performed in the Outpatient Pneumology Clinic of The Medical University of Lodz. It has been approved by the Bioethical Committee of the Medical University of Lodz (No. RNN/58/21/KE). Participants recruited for the study between December 2020 and May 2021 were patients after interstitial COVID 19 pneumonia either leaving the pneumology unit of Barlicki Memorial Teaching Hospital of the Medical University of Lodz or patients visiting the outpatient pneumology clinic of Teaching Hospital No. 1 up to six weeks after discharge from other centers. Participants were equipped with telemedicine equipment consisting of pulse oximeters (MIR Spirotel ${ }^{\circledR}$ ) connected with Samsung Galaxy Tab 3 tablets; the set was equipped with an internet SIM card and associated with a web application Atencare (Mediguard $\circledast$ ). Everyone underwent the 15-minute technical training and informed consent was obtained from all subjects. Every day the participants were supposed to evaluate their percutaneous saturation and heart rate with a minimum of 2-minute measurement; performed at rest with no oxygen supplementation for at least 15 minutes. The intensity of cough and dyspnea was estimated in simple 1 to 5 points daily surveys. The results were analyzed daily and participants were able to reach the physician-in-charge at any time with a phone call to evaluate new/worsening symptoms and assess the urgency of medical intervention.

At the same time, the clinical examination of participants was performed twice; first between two to six weeks after hospital discharge and second after two consecutive months. Apart from physical 
examination, pulmonary function tests (PFTs) such as spirometry, lung transfer for carbon dioxide $\left(T_{L, C O}\right)$, and 6-minute walk test (6MWT), have been performed. Every patient's radiological changes were assessed in an initial and controlled radiographic study; the choice of a second one was individual (highresolution computed tomography, computed tomography angiography, chest X-ray). CT scans were assessed by two individual experts with a minimum of 3-years' experience in lung tomography. All methods were performed in accordance with relevant guidelines and regulations, including Declaration of Helsinki.

Statistical analysis was performed using R software for MacOS. Continuous data were presented as the mean with SD or median with interquartile range (IQR), depending on the distribution of data. Variables were compared using the unpaired Student's t-test, Welch t-test, or the Wilcoxon rank-sum test with continuity correction, depending on data normality and homogeneity of variance. Categorical data were analyzed using the Chi-square test or Fisher's Exact Test, based on assumptions of the tests.

\section{Results}

Thirty patients of the mean age 55 (mean 55.23; SD +/- 10.64 years) were involved in the study. Participants' data are presented in Table 1. The group was divided according to clinical improvement defined as the $\geq 10 \%$ functional vital capacity (FVC) raise or $\geq 15 \%$ lung transfer for carbon monoxide $\left(T_{L, C O}\right)$ rise. Our findings suggest that at-rest home saturation measurements below $94 \%$ $(p=0.03)$ correspond with the lack of clinical improvement in post-COVID observation $(p=0.03)$. The nonimprovement group presented with lower mean - 94 (93-96) \% vs 96 (95-97)\%, p=0.01 and minimum saturation - $89(86-92) \%$ vs. $92(90-94) \%, p=0.04$. They also presented higher variations in saturation measurements; saturation amplitude was $9(7-11) \%$ vs $7(4-8) \%, p=0.03$; up to day 22 most of the saturation differences reached statistical significance. Last but not least, we discovered that participants missing 2 or more measurements during the observation were more often ranked into the clinical improvement group $(p=0.01)$. Heart rate day-to-day measurements did not differ between both groups; gathered data about dyspnea and cough intensity did not reach statistical significance either.

\section{Discussion}

Telesolutions have been increasingly developing during the past epidemics, e.g. for contact investigations and disease control [6]. In the time of the Ebola outbreak between the years 2014 to 2016, a mobile application helped to trace and monitor confirmed cases [7], increasing time to case registration, completeness, and security of the data. When SARS CoV 1 was dealt with in Taiwan, online communication via a webcam increased the availability of medical consultations and reduced their costs [8]. Swiss teleservice notifications associated with fever were proven to reflect influenza activity [9].

Lately, the pandemic situation forced outpatient care out of the doctor's office. Italian authors, like Omboni [10], complained of insufficient telemedicine implementation during the first striking COVID 19 wave and suggested it is a must in a modern health care system, especially in terms of chronically-ill 
patients during a lockdown. Nevertheless, more attention in the literature has been put on telehealth concerning home-isolated COVID 19 patients and controlling their disease [11], especially after the unpredictable character of SARS CoV 2-associated pneumonia presented itself throughout the world. The China health center[12] has set a network for COVID 19 alert and response with 126 network hospitals involved. Between January 28 and February 17 in 202063 teleconsulted patients had severe pneumonia alongside 591 were moderate cases. At that time, there were mobile devices used for collecting, evaluating, and reporting patient vital signs to the caring team in isolation wards. The authors outline that the bedside system allowed limiting the exposure to patients' contagious secretions and the communication system was utilized to build and remotely train multidisciplinary health teams for a more comprehensive treatment. An utterly different telesurveillance solution for home-treated patients based on questionnaires in a mobile application has been developed by a French group [13], with more than 65000 users in Paris and its core based on Medical Responders and physicians reacting to changing subjective health status of ill individuals.

The effects of telemedicine implementation and its impact are well known in cardiology, with trials such as the TIM-HF2 [14] which demonstrated that remote telemonitoring reduced days lost due to unplanned cardiovascular hospitalizations, as well as it documented a reduction in all-cause mortality among patients managed in the study. In chronic obstructive pulmonary disease, our research group has previously shown that a decrease in saturation exceeding $4 \%$ can predict an exacerbation in the forthcoming 7 days [15]. With enough time and training, home spirometry starts to correlate with hospital spirometry among the idiopathic pulmonary fibrosis group [16]. Among the less obvious effects of telemedicine-enhanced home care, the worth-to-consider effects are both improved psychological wellbeing and individually tailored treatment decisions.

Much is known about the average recovery after COVID 19 pneumonia and persistent symptoms; however, the variety of research does not answer the question of which patients should be supervised with special attention. As to our knowledge, no other group monitored patients at home for a longer period than we managed to do. This study was aimed to stratify the usefulness of day-to-day saturation and heart rate measurements, as well as the subjective extent of dyspnea and cough, in the post-COVID care among the previously hospitalized survivors. Our findings suggest that a patient who provides at-rest saturation measurements lower than $94 \%$ will not significantly improve in pulmonary function tests - FVC and DLCO - after 2 to 3 months post-discharge. This equals continuous exercise intolerance.

Most of the existing research associated with COVID 19 and telehealth consider the acute infection stage, sometimes with a short sequence afterwards. Motta et al. monitored saturation, heart rate, body temperature and peak expiratory flow of 12 patients during 30 consecutive days of acute home-treated individuals with mild to asymptomatic SARS CoV 2 infestations to evaluate a quick response system during the worsening symptoms[17]. The findings have shown significant decrease in SpO2 and increase in heart rate during the illness, while PEF values dropped below $80 \%$ of normal range among 4 of the participants. The authors outlined that there was not enough data to guide the use of home pulse oximetry or validate it in disease progression. 
O'Carroll et collaborates used remote oxygen saturation monitoring in COVID-19 cases to facilitate discharge of non-oxygen dependent patients and have their safe follow-u[18]. During the median time of 12 days of measurements, the telemonitoring allowed to detect $3 / 18$ patients with desaturations because of worsening COVID 19 infiltrates and 1/18 worsening from hospital-acquired pneumonia developing after the hospital discharge. Telemedicine served its role - it allowed managing patients' conditions in a more controlled manner; no one from this group (4/18) required non-invasive or invasive ventilation during readmission. Of note, the alerts that lead to medical attention were programmed to be generated after every measurement lower than $94 \%$ Sp02, consistent with our findings. The frequency of measurements was higher (mean 3.9 vs 5.7 per day) in the readmission group[18], which is harmonious with our observations concerning a higher cooperation rate among participants with more severe diseases. Similar research from Grutters et al. proved the 5-day (+/-3.8) shortening of hospital stay among the 33 participants group via the use of telehealth. It also allowed a safe follow-up with 3 readmissions and 1 pulmonary embolism diagnosed, along with the cost-effectiveness of a whole system. Most patients in these studies rate telemonitoring to be friendly and useful.

Research by Martínez-García et al. included two groups of patients in the surveillance[19]: 224 outpatients traced from the beginning of the disease and 89 inpatients after discharge. Every patient sent oxygen saturation and temperature 3 times a day. Proactively, the patient was reached at least once a day. Until the termination of the study after 30 days, 38 (16.90\%) outpatients were referred to the Emergency Department, 18 were hospitalized (8.03\%), and 2 deceased. One patient from the inpatient group was re-hospitalized and one left the study. Importantly, neither deaths nor a vital emergency happened at home. The average time of monitoring was 11.64 (+/-3.58) days, and $224(73.68 \%)$ patients were discharged during the 30 days of study.

Patients are reluctant to participate in telehealth research for various reasons explored e.g. by Sanders et al. [20]. Most anxiety comes from technical requirements - which are often misunderstood and exaggerated. There is a group of patients that consider telemedical surveillance with a high degree of dependency and ill health, which is unbearable to them. At the same time, others are glad to have their current health care providers and they are hesitant about the care methods they are unfamiliar with. Great telemonitoring adherence data comes from the paper by Lang et al. [21]. Among the analyzed group, some participants withdrew from the study during its course - referred to as the drop-outs. 41 patients gave reasons for dropping out after a period of sending data. They can be further categorized into groups: no perceived benefits for health; no need for telemonitoring; investing too much time in the participation; insufficient user-friendliness; feeling a loss of privacy. The most mentioned reasons for dropping out were no perceived benefit $(19 / 41 ; 46.3 \%)$ and the lack of telemonitoring needs $(18 / 41$; 43.9\%). Cook [22] also outline that the majority of users resigning from telehealth did not find the equipment useful once they had tried it, while Foster's[23] telehealth engagement study reported that as much as $40.1 \%(n=2852)$ of decliners did not feel a need for additional health support, $27.2 \%(n=1932)$ stated being too busy to use it and $15.3 \%(n=1092)$ of decliners were not interested in the research. This data is greatly consistent with our findings, where non-adherence and omitting the daily measurements are correlated with functional improvement after COVID-19. We did not investigate participants' 
motivation though, so we can only speculate that they did not feel the necessity to stay under strict surveillance.

Compared to the research cited above, our prospective study is unique because we prolonged the monitoring until a minimum of 2 weeks of SpO2 >=95\% with a mean observation time of 67 (range: 45114) days. The program allowed us to notice serious events in patients' individual post-COVID history. It becomes crucial when you realize a striking study by Chopra et al. [3] who depicted that from 1250 COVID 19 survivors in the US State - Michigan - 84 patients (6.7\% of hospital survivors and $10.4 \%$ of ICU-treated hospital survivors) died in the following 60 days, bringing the overall mortality rate for the cohort to $29.2 \%$ hospitalized and $63.5 \%$ of treated in ICUs. Data from Bellan et al. further confirm these results with $5 \%$ post-discharge 30-day mortality[24]. Furthermore, 189 convalescents (15.1\%) became re-hospitalized in the same period. From our group, one of the patients returned to a hospital during the study because of Clostridioides difficile diarrhea as a post-hospitalization and post-antibiotic consequence. At the time of observation, the additional diagnoses were: one outpatient post-COVID pulmonary embolism; one hereditary thrombophilia (in another person); two asthma diagnoses; urinary bladder ulcers of possible viral etiology in one patient; myocarditis (two suspected and one of them confirmed in the MRI). There was also one underlying interstitial lung disease suspected but the final diagnosis of severe emphysema with overlapping post-COVID radiological changes has been determined. None of the participants died or had rapidly worsening respiratory parameters. A few were hospitalized in pulmonary rehabilitation units, with notable subjective improvement; the efficacy of pulmonary rehabilitation is often confirmed in the research papers [25].

On the other hand of the statistically important data, the heart rate measurements did not prove to be useful in real-life telemonitoring study, probably because compensatory tachycardia was deeply modified by the use of medications like $\beta$-blockers and ivabradine. We were also disappointed with dyspnea and cough self-assessment scales that did not correspond with pulmonary improvement. Interestingly, it appears from existing studies that there is no significant difference in PFTs when comparing patients with persistent COVID-19-related symptoms and asymptomatic ones [26].

The main limitation of our research is no randomization; every patient hospitalized for COVID 19 pneumonia was allowed to join it. The starting point slightly differed between patients hospitalized in our unit and those from other centers, forming a possible bias. 6-minute walk tests were performed with different supervisors and it probably had an impact on patients' engagement in the test itself. The severity of interstitial pneumonia among participants was not equal and men were predominant. The substantial benefits come from a longer observation time and addressing the pulmonary function tests to pulse oximetry results; as far as we are concerned no other researchers found such a correlation. Every patient had technical training with access to technical and medical consultation whenever problems occurred; just to eliminate loss of data or potentially hazardous events.

\section{Conclusions}


A better understanding of the disease's natural history will ultimately lead us to a better understanding of long COVID symptoms and corresponding threats. In this paper, we have found home oxygen saturation telemonitoring to be useful in the prediction of the trajectory of the disease course. Our findings suggest that detection of at-rest home saturation measurement equal to or below $94 \%$ corresponds with the lack of clinical improvement in the time of observation and this group of patients presented higher variability of day-to-day oxygen saturation measurements. The determination of which patient should be involved in telemedicine programs after discharge requests further research.

\section{Declarations}

\section{Authors' contributions}

$\mathrm{KB}$ acts as the main researcher and executor of this work with a major role in the study conception, design, data acquisition, analyzis and interpretation of the data. KB wrote a draft of the manuscript. AJB, JMD and WJP were all mutual designers of the study and interpreted the data. JMD was involved in the acquisition of the data. AJB coanalyzed the data, while AJB, JMD, WJP contributed to its interpretation. $K B, A J B, J M D$, WJP revised the work, approved the submitted version and they agree to be personally accountable for all aspects of the work.

\section{Acknowledgments}

We want to honor Waldemar Obrebski who is a research team member helping in acquiring the research data. We would also like to thank all of the participating patients, as well as the students engaged in the process of patient care in the outpatient clinic of Barlicki Memorial Hospital.

\section{Competing interests}

The author(s) declare no competing interests.

\section{Funding}

This research was not supported by any specific funding.

\section{References}

1. Portnoy J, Waller M, Elliott T. Telemedicine in the Era of COVID-19. J Allergy Clin Immunol Pract. 2020;8:1489-91.

2. Leite VF, Rampim DB, Jorge VC, de Lima M do CC, Cezarino LG, da Rocha CN, et al. Persistent Symptoms and Disability After COVID-19 Hospitalization: Data From a Comprehensive Telerehabilitation Program. Arch Phys Med Rehabil. 2021;102:1308-16.

3. Chopra V, Flanders SA, O'Malley M, Malani AN, Prescott HC. Sixty-Day Outcomes Among Patients Hospitalized With COVID-19. Ann Intern Med. American College of Physicians; 2021;174:576-8. 
4. Abdelnabi M, Leelaviwat N, Eshak N, Mekraksakit P, Nugent K, Payne JD. COVID-19 discharge and follow-up recommendations. Proc Bayl Univ Med Cent. 34:73-5.

5. Telemedicine I of M (US) C on ECA of, Field MJ. Introduction and Background [Internet]. Telemed. Guide Assess. Telecommun. Health Care. National Academies Press (US); 1996 [cited 2021 Nov 1]. Available from: https://www.ncbi.nlm.nih.gov/books/NBK45440/

6. Keshvardoost S, Bahaadinbeigy K, Fatehi F. Role of Telehealth in the Management of COVID-19: Lessons Learned from Previous SARS, MERS, and Ebola Outbreaks. Telemed J E-Health Off J Am Telemed Assoc. 2020;26:850-2.

7. Reducing Ebola Virus Transmission: Improving Contact Tracing in Sierra Leone [Internet]. Innov. Poverty Action. 2015 [cited 2021 Dec 10]. Available from: https://www.povertyaction.org/study/reducing-ebola-virus-transmission-improving-contact-tracing-sierra-leone

8. Chang T, Lee J, Wu S. The telemedicine and teleconsultation system application in clinical medicine. Conf Proc Annu Int Conf IEEE Eng Med Biol Soc IEEE Eng Med Biol Soc Annu Conf. 2004;2004:33925 .

9. Blozik E, Grandchamp C, von Overbeck J. Influenza surveillance using data from a telemedicine centre. Int J Public Health. 2012;57:447-52.

10. Omboni S. Telemedicine During the COVID-19 in Italy: A Missed Opportunity? Telemed J E-Health Off J Am Telemed Assoc. 2020;26:973-5.

11. Ohannessian R, Duong TA, Odone A. Global Telemedicine Implementation and Integration Within Health Systems to Fight the COVID-19 Pandemic: A Call to Action. JMIR Public Health Surveill. 2020;6:e18810.

12. Zhai $Y$, Wang $Y$, Zhang $M$, Gittell JH, Jiang S, Chen B, et al. From Isolation to Coordination: How Can Telemedicine Help Combat the COVID-19 Outbreak? [Internet]. 2020 Feb p. 2020.02.20.20025957. Available from: https://www.medrxiv.org/content/10.1101/2020.02.20.20025957v1

13. Yordanov Y, Dechartres A, Lescure X, Apra C, Villie P, Marchand-Arvier J, et al. Covidom, a Telesurveillance Solution for Home Monitoring Patients With COVID-19. J Med Internet Res. 2020;22:e20748.

14. Koehler F, Koehler K, Deckwart O, Prescher S, Wegscheider K, Kirwan B-A, et al. Efficacy of telemedical interventional management in patients with heart failure (TIM-HF2): a randomised, controlled, parallel-group, unmasked trial. The Lancet. Elsevier; 2018;392:1047-57.

15. Miłkowska-Dymanowska J, Białas AJ, Obrębski W, Górski P, Piotrowski WJ. A pilot study of daily telemonitoring to predict acute exacerbation in chronic obstructive pulmonary disease. Int J Med Inf. 2018;116:46-51.

16. Moor CC, Mostard RLM, Grutters JC, Bresser P, Aerts JGJV, Chavannes NH, et al. Home Monitoring in Patients with Idiopathic Pulmonary Fibrosis. A Randomized Controlled Trial. Am J Respir Crit Care Med. American Thoracic Society - AJRCCM; 2020;202:393-401.

17. Motta LP, da Silva PPF, Borguezan BM, do Amaral JLM, Milagres LG, Bóia MN, et al. An emergency system for monitoring pulse oximetry, peak expiratory flow, and body temperature of patients with 
COVID-19 at home: Development and preliminary application. PLoS ONE. 2021;16:e0247635.

18. O'Carroll O, MacCann R, O'Reilly A, Dunican EM, Feeney ER, Ryan S, et al. Remote monitoring of oxygen saturation in individuals with COVID-19 pneumonia. Eur Respir J. 2020;56:2001492.

19. Martínez-García M, Bal-Alvarado M, Santos Guerra F, Ares-Rico R, Suárez-Gil R, Rodríguez-Álvarez A, et al. Monitoring of COVID-19 patients via telemedicine with telemonitoring. Rev Clin Esp. 2020;220:472-9.

20. Sanders C, Rogers A, Bowen R, Bower P, Hirani S, Cartwright M, et al. Exploring barriers to participation and adoption of telehealth and telecare within the Whole System Demonstrator trial: a qualitative study. BMC Health Serv Res. 2012;12:220.

21. Lang C, Voigt K, Neumann R, Bergmann A, Holthoff-Detto V. Adherence and acceptance of a homebased telemonitoring application used by multi-morbid patients aged 65 years and older. J Telemed Telecare. SAGE Publications; 2020;1357633X20901400.

22. Cook EJ, Randhawa G, Sharp C, Ali N, Guppy A, Barton G, et al. Exploring the factors that influence the decision to adopt and engage with an integrated assistive telehealth and telecare service in Cambridgeshire, UK: a nested qualitative study of patient 'users' and 'non-users.' BMC Health Serv Res. 2016;16:137.

23. Foster A, Horspool KA, Edwards L, Thomas CL, Salisbury C, Montgomery AA, et al. Who does not participate in telehealth trials and why? A cross-sectional survey. Trials. 2015;16:258.

24. Bellan M, Soddu D, Balbo PE, Baricich A, Zeppegno P, Avanzi GC, et al. Respiratory and Psychophysical Sequelae Among Patients With COVID-19 Four Months After Hospital Discharge. JAMA Netw Open. 2021;4:e2036142.

25. Zhu P, Wang Z, Guo X, Feng Z, Chen C, Zheng A, et al. Pulmonary Rehabilitation Accelerates the Recovery of Pulmonary Function in Patients With COVID-19. Front Cardiovasc Med. 2021;8:691609.

26. Munker D, Veit T, Barton J, Mertsch P, Mümmler C, Osterman A, et al. Pulmonary function impairment of asymptomatic and persistently symptomatic patients 4 months after COVID-19 according to disease severity. Infection. 2021;1-12.

\section{Table}

Table 1. Characteristics of study participants. Abbreviations: $B M I-$ body mass index; $C T$ - computed tomography; FEV1 - forced expiratory volume in 1 second; FVC - forced vital capacity; TLC - total lung capacity; $T_{L, C O}$ transfer factor of the lung for carbon monoxide 


\begin{tabular}{|ll|}
\hline & Value \\
\hline Parameter & \\
\hline Male, $n$ (\%) & $55.23(10.64)$ \\
\hline BMI, kg/m2, mean (SD) & $23 / 30(76.66)$ \\
\hline Packyears, median (IQR) & $28.85(5.12)$ \\
\hline Hospitalization days, median (IQR) & $15(8-30)$ \\
\hline CT initial lung involvement 0-4 scale, points (IQR) & $12(7-26.25)$ \\
\hline Mean initial saturation, \%, mean (SD) & $3(2-4)$ \\
\hline Mean initial heart rate, bpm, mean (SD) & $95.53(1.79)$ \\
\hline Mean initial dyspnea, 1-5 visual scale, points, mean (SD) & $78.48(8.62)$ \\
\hline Mean initial cough, 1-5 visual scale, points (SD) & $1.70(0.70)$ \\
\hline FEV1, L, mean (SD) & $1.43(0.62)$ \\
\hline FEV1, \%, mean (SD) & $2.75(1.01)$ \\
\hline FVC, L, mean (SD) & $78.15(20.36)$ \\
\hline FVC, \%, mean (SD) & $3.36(1.32)$ \\
\hline TLC, L, median, (IQR) & $75(21.27)$ \\
\hline TLC, \%, mean, (SD) & $5.04(4.24-6.75)$ \\
\hline TL,co, mL/min/mm Hg, mean, (SD) & $79.96(24.93)$ \\
\hline TL,Co, \%, mean, (SD) & $6.27(2.72)$ \\
\hline
\end{tabular}

